\title{
Retrospective Review of MET Gene Mutations
}

\author{
Maryam Zenali ${ }^{1 *}$ and James deKay ${ }^{2 *}$, Zesheng Liu ${ }^{3}$, Stanley Hamilton ${ }^{1}$, \\ Zhuang Zuo ${ }^{4}$, Xinyan Lu ${ }^{5}$, Rania Bakkar ${ }^{6}$, Gordon Mills ${ }^{3}$, Russell Broaddus ${ }^{1}$ \\ ${ }^{1}$ Department of Pathology and Laboratory Medicine, University of Texas MD Anderson Cancer Center, Houston, Texas \\ 2Department of Pathology and Laboratory Medicine, University of Vermont Medical Center, Burlington, Vermont \\ ${ }^{3}$ Institute of Personalized Cancer Therapy, University of Texas MD Anderson Cancer Center, Houston, Texas \\ ${ }^{4}$ Department of Molecular Pathology, University of Texas MD Anderson Cancer Center, Houston, Texas \\ ${ }^{5}$ Department of Hematopathology, University of Texas MD Anderson Cancer Center, Houston, Texas \\ ${ }^{6}$ Department of Pathology, University of New Mexico, Albuquerque, New Mexico \\ *Both Drs. Zenali and deKay are co-first authors
}

Correspondence to: Maryam Zenali, email: maryam.zenali@uvmhealth.org

Keywords: C-MET, co-mutations, FISH, targeted therapy

Received: February 18, $2015 \quad$ Accepted: May 05, $2015 \quad$ Published: May 14, 2015

This is an open-access article distributed under the terms of the Creative Commons Attribution License, which permits unrestricted use, distribution, and reproduction in any medium, provided the original author and source are credited.

\section{ABSTRACT}

C-MET proto-oncogene is a tyrosine kinase situated on chromosome 7. C-MET and its ligand hepatocyte growth factor/scatter factor (HGF/SF) play a role in proliferation, differentiation and organ development. C-MET genetic aberrations are found associated with driving tumorigenesis. In this retrospective study, we reviewed molecular analysis data gathered from a cancer institute during a two-year period (2010-2012). Upon detection of tumors harboring c-MET mutations, we determined the status of the other mutations tested and evaluated c-MET expression by fluorescent in-situ hybridization (FISH). Our search resulted in identification of $134 \mathrm{c}-$ MET mutations, $44 \%$ of which had mutations of at least one of the other genes tested. No c-MET expression aberrancy was detected in this subset by FISH. Survival amongst the patients with surgically resected metastatic colorectal cancers (CRC) was slightly better in those with only a C-MET mutation compared to those with no mutation detected, although the difference was not statistically significant. When c-MET inhibition becomes an integrated part of chemotherapy practice, our observed frequency of co-mutations will be an argument for utilizing c-MET targeted treatment in combination with other targeted drugs and therapeutic strategies. Larger studies can aid to further parse out c-MET prognostic and therapeutic significance.

\section{INTRODUCTION}

C-MET tyrosine kinase was first discovered in the1980s [1]. C-MET and its ligand HGF/SF are essential cellular components during embryogenesis, morphogenesis, and development of organs such as liver, skeletal muscle, and placenta. C-MET aberrations at the genetic level or post-translational overexpression of the receptor have been studied in many tumors; association of these alterations with oncogenesis and tumor progression has been proposed [1].

C-MET, as detailed in a study by Ma et. al, contains 3 main structural domains: an extracellular Sema domain (Exon 2), a juxtamembrane domain (Exons 14/15) and a tyrosine kinase domain where many of the c-MET mutations have been reported [2]. Activation of c-MET is achieved via $\mathrm{HGF} / \mathrm{SF}$ binding which results in a variety of actions depending on the cellular context, varying from proliferation, motility and differentiation in wild type cells to invasion and metastasis in neoplastic cells. In tyrosine kinase pathways where c-MET is a component, ligand binding precedes receptor dimerization. Mutations in these or associated genes circumvent the appropriate ligand dependent regulation. C-MET activation can occur by amplification/ overexpression, activating point mutations, paracrine signaling or over expression of its ligand hepatocyte growth factor/scatter factor [1-4].

C-Met signaling has been implicated in a variety of malignancies, including gastrointestinal, genitourinary, breast, gynecologic, pulmonary, head and neck cancers and melanoma. Its genetic aberrations have been reported in a 
small percentage of this wide variety of tumors $[1,5]$. Certain c-MET mutations are found to be germline with unknown transforming activity while others are implicated in driving neoplasia.

Availability HGF/SF-MET inhibitors with a range of potencies and specificities has provided a base for assessing their therapeutic value, and initial clinical trials have shown benefit from these treatments in several tumor types. Since c-MET signaling converges with a multitude of pathways, it can lead to activation of several downstream effectors [5]. This diversity substantiates the efforts of c-MET targeting, but biochemical crosstalk between pathways with potential neoplastic rescue process argues for combination chemotherapy.

In this retrospective study we reviewed molecular diagnostic data resulting from a series of mutational analysis during the 2010-2012 period in a cancer center institution. The primary objective was to gain insight into patterns of c-MET mutations and patterns and frequency of comutations. We also compared survival outcome in metastatic colorectal adenocarcinoma between those harboring only c-MET mutations as opposed to those with no mutations detected on a 14-gene test.

\section{MATERIALS AND METHODS}

We reviewed the molecular analysis report from over 2000 solid tumors undergone mutation testing at MD Anderson during 2010-2012. Cases (except a small subset of primary brain tumors) were predominantly high stage solid tumors where the mutational analysis was performed to assess for considerations of targeted additional therapy. These tests included a custom designed mutation analysis panel as described previously (Leuk Lymphoma 54(1):138-44; 2013). This quantitative primer-extension MALDI-TOF assay (PE-MALDI) interrogated a total of 88 hotspot mutations in 11-genes (AKT-1, BRAF, GNAQ, GNAS, IDH1, IDH2, KRAS, MET, NRAS, PIK3CA, RET). Mutation detection was carried out utilizing a 9 -well based 11-gene mutation hotspot screen developed by our laboratory using Massarray ${ }^{\circledR}$ platform (Sequenom, San Diego, CA). Briefly, DNA was PCR amplified and subjected to single base-primer extension using iPLEX Gold kit and analyzed on MALDI-TOF mass spectrometry (Sequenom, San Diego, CA). The regions of interest were PCR amplified using 10ng of DNA per well. Subsequently PCR products were treated with shrimp alkaline phosphatase to dephosphorylate unincorporated nucleotides followed by locus specific single base extension with mass modified di-deoxy nucleotides (ddNTP).

Primers for both PCR amplification and single-base extension were designed by Sequenom's MassARRAY Designer software and obtained from Integrated DNA Technologies (IDT, Coralville, IA). The mass of the products of single-base extension were analyzed by MALDI-TOF for SNP detection. In addition, the exons 18 to 21 of the EGFR gene, exons 11, 13, and 17 of the KIT gene and in a subset of cases, the exons 4 to 9 of the TP53 gene were also analyzed in each sample by Sanger sequencing. In all cases, DNA was obtained from unstained formalin fixed paraffin embedded (FFPE) tissue sections using the PicoPure DNA Extraction Kit (Life Technologies, Grand Island, NY) following manufactures instructions. In the c-MET mutated subset, patient's demographics, tumor types and stage, SNP patterns, their distribution amongst tumor types, secondary tumors and incidences of co-mutations were recorded (Tables 1-3). Metastatic CRC was the largest tumor category harboring c-MET mutation, in which we compared survival outcome of patients with only c-MET mutation to those with no detectable mutation using Kaplan Meier method and difference compared by log-rank. In the same subset of patients we evaluated whether c-MET mutational status showed associations with age and/or gender by logistic regression analysis.

Additionally, we reviewed the c-MET FISH results when available, to examine whether there is an association of MET amplification with presence of genetic aberration. MET FISH was performed in the clinical cytogenetics diagnostic lab at MD Anderson Cancer center, following the standard clinical procedure established in the laboratory. In brief, glass slides from formalin-fixed paraffin embedded tissue (FFPE) are pre-treated and hybridized with the MET and chromosome 7-centromere FISH probe mixture (Poseidon/Empire Genomics, Buffalo, NY). FISH results were scored and reviewed on a fluorescence microscope. A total of 60 cells were scored. MET amplification was considered positive if the ratio of average MET to average of CEN 7 was equal or greater than 2.0 or more than 20 copies of MET signals with clusters noted in $>10 \%$ of the cells.

\section{RESULTS}

Upon review of the molecular data, we found 134 c-MET mutations, involving 133 tumors (2010 -2012), which correspond to $6-7 \%$ of the total number of tumors. 5 different c-MET point mutations were detected by DNA sequencing (Table 1). The majority of mutations were observed in codon 375.81 cases had Asn to Ser mutation at codon 375 (N375S), corresponding to $60 \%$. 38 cases had a Thr to Ile mutation at codon 1010 (T1010I), corresponding to $28 \%$. 13 cases had an Arg to Cys at codon 988 (R988C), corresponding to $10 \%$. One case had a Tyr to His mutation at codon 1248 $(\mathrm{Y} 1248 \mathrm{H})$, corresponding to $0.7 \%$, and one had a Tyr to Asp at codon 1253 (Y1253D), corresponding to $0.7 \%$. One case of melanoma (Lentigo Maligna type/stage IV disease) had 2 simultaneous c-MET mutations (N375S and T1010I). FISH assay for c-MET gene copy number amplification was carried in $46 \%$ of the c-MET mutated (62/134), all of which were negative.

$44 \%$ (59 of 134) cases with c-MET mutation had mutations of at least one of the other 13 genes in the tested (10 additional genes by Sequenom and 3 by Sangers) with $9 \%$ (12 of 134) having 2 or more co-mutations. These co- 


\begin{tabular}{|c|c|c|c|c|c|}
\hline $\begin{array}{l}\text { Tumor type (with } \\
\text { c-MET mutation) }\end{array}$ & $\begin{array}{l}\text { Other tumors in } \\
\text { these patients }\end{array}$ & $\begin{array}{l}\text { Patients' age/ } \\
\text { sex }\end{array}$ & $\begin{array}{l}\text { Tumor } \\
\text { stage }\end{array}$ & $\begin{array}{l}\text { Mutation } \\
\text { exon (s) }\end{array}$ & $\begin{array}{l}\text { Mutation codon (s)/aa } \\
\text { change (s) }\end{array}$ \\
\hline $\begin{array}{l}\text { Colorectal Adenoca. } \\
\mathrm{n}=43 \\
\% \text { mutated: } 9.5 \%\end{array}$ & $\begin{array}{l}\text { Melanoma } \times 1 \\
\text { Prostatic } \mathrm{Ca} \times 1\end{array}$ & $\begin{array}{l}\text { Median age: } 59 \\
\text { Avg age: } 56.4 \\
\mathrm{M} / \mathrm{F}=28 / 15\end{array}$ & $\begin{array}{l}\text { IV: } 28 \\
\text { III: } 11 \\
\text { II: } 4\end{array}$ & $\begin{array}{c}2 \\
14 \\
14 \\
19 \\
19\end{array}$ & $\begin{array}{l}\text { N375S } \times 19 / \text { Asn to Ser } \\
\text { T1010I } \times 18 / \text { Thr to Ile } \\
\text { R988C } \times 4 / \text { Arg to Cys } \\
\text { Y1253D } \times 1 / \text { Tyr to Asp } \\
\text { Y1248H } \times 1 / \text { Tyr to His }\end{array}$ \\
\hline $\begin{array}{l}\text { Melanoma } \\
\mathrm{n}=14 \\
\% \text { mutated: } 5.5 \%\end{array}$ & $\begin{array}{l}\text { BCC \& SCC } \times 4 \\
\text { Dysplastic nevus } \times 2 \\
\text { PTC } \times 1 \\
\text { Prostatic Ca } \times 1\end{array}$ & $\begin{array}{l}\text { Median age: } 62 \\
\text { Avg age: } 59 \\
M / F=10 / 4\end{array}$ & $\begin{array}{l}\text { IV: } 13 \\
\text { III: } 1\end{array}$ & $\begin{array}{c}2 \\
14 \\
14\end{array}$ & $\begin{array}{l}\text { N375S } \times 10 / \text { Asn to Ser } \\
\text { R988C } \times 2 / \text { Arg to Cys } \\
\text { T1010I } \times 3 / \text { Thr to Ile } \\
* \text { one tumor had } 2 \mathrm{cMET} \\
\text { mutations }\end{array}$ \\
\hline $\begin{array}{l}\text { Gastric Adenoca. } \\
\mathrm{n}=2 \\
\% \text { mutated: } 25 \%\end{array}$ & & $\begin{array}{l}51 \mathrm{y} / \mathrm{o} \mathrm{F} \& \\
69 \mathrm{y} / \mathrm{o} \mathrm{F}\end{array}$ & $\begin{array}{l}\text { III: } 1 \\
\text { II: } 1\end{array}$ & 2 & N375S $\times 2 /$ Asn to Ser \\
\hline $\begin{array}{l}\text { Appendiceal Adenoca. } \\
\mathrm{n}=2 \\
\% \text { mutated: } 13 \%\end{array}$ & & $\begin{array}{l}55 \mathrm{y} / \mathrm{o} \mathrm{F} \& \\
33 \mathrm{y} / \mathrm{o} \mathrm{F}\end{array}$ & IV: 2 & $\begin{array}{c}2 \\
14\end{array}$ & $\begin{array}{l}\text { N375S } \times 1 / \text { Asn to Ser } \\
\text { R988C } \times 1 / \text { Arg to Cys }\end{array}$ \\
\hline $\begin{array}{l}\text { Hepatocellular } \mathrm{Ca} \\
\mathrm{n}=2 \\
\% \text { mutated: } 17 \%\end{array}$ & & $\begin{array}{l}62 \mathrm{y} / \mathrm{o} \mathrm{M} \& \\
72 \mathrm{y} / \mathrm{o} \mathrm{M}\end{array}$ & $\mathrm{N} / \mathrm{A}$ & $\begin{array}{c}2 \\
14\end{array}$ & $\begin{array}{l}\text { N375S } \times 1 / \text { Asn to Ser } \\
\text { R988C } \times 1 / \text { Arg to Cys }\end{array}$ \\
\hline $\begin{array}{l}\text { Duodenal Adenoca. } \\
\mathrm{n}=1 \\
\% \text { mutated: } 7 \%\end{array}$ & & 44 y/o F & IV: 1 & 2 & N375S $\times 1 /$ Asn to Ser \\
\hline $\begin{array}{l}\text { Pancreatic Adenoca. } \\
\mathrm{n}=1 \\
\% \text { mutated: } 8.3 \%\end{array}$ & & 64 y/o M & IV: 1 & 2 & N375S $\times 1 /$ Asn to Ser \\
\hline $\begin{array}{l}\text { Lung Adenoca. } \\
\mathrm{n}=8 \\
\% \text { mutated: } 4.8 \%\end{array}$ & $\begin{array}{l}\mathrm{SCC} \times 1 \\
\text { Prostatic } \mathrm{Ca} \times 1\end{array}$ & $\begin{array}{l}\text { Avg age: } 64 \\
\text { Median age: } 65 \\
\text { M/F: } 4 / 4\end{array}$ & $\begin{array}{l}\text { IV: } 7 \\
\text { N/A: } 1\end{array}$ & $\begin{array}{c}2 \\
14 \\
14 \\
\end{array}$ & $\begin{array}{l}\text { N375S } \times 6 / \text { Asn to Ser } \\
\text { R988C } \times 1 / \text { Arg to Cys } \\
\text { T1010I } \times 1 / \text { Thr to Ile }\end{array}$ \\
\hline $\begin{array}{l}\text { Thyroid Papillary } \mathrm{Ca} \\
\mathrm{n}=3 \\
\% \text { mutated: } 12 \%\end{array}$ & Lung Adenoca. $\times 1$ & $\begin{array}{l}\text { Avg age: } 68 \\
\text { Median age: } 65 \\
\text { M/F: N/A } \\
\end{array}$ & IV: 3 & 2 & N375S ×3/Asn to Ser \\
\hline $\begin{array}{l}\text { Thyroid Medullary } \mathrm{Ca} \\
\mathrm{n}=1 \\
\% \text { mutated: } 50 \%\end{array}$ & & $60 \mathrm{y} / \mathrm{o} \mathrm{M}$ & $\mathrm{N} / \mathrm{A}$ & 14 & T1010I $\times 1 /$ Thr to Ile \\
\hline $\begin{array}{l}\text { Ewing Sarcoma } \\
\mathrm{n}=1 \\
\% \text { mutated: } 33 \% \\
\end{array}$ & & $25 \mathrm{y} / \mathrm{o} \mathrm{F}$ & $\mathrm{N} / \mathrm{A}$ & 14 & T1010I $\times 1 /$ Thr to Ile \\
\hline $\begin{array}{l}\text { Prostatic Adenoca. } \\
\mathrm{n}=1 \\
\% \text { mutated: } 10 \%\end{array}$ & & $67 \mathrm{y} / \mathrm{o} \mathrm{M}$ & IV & 2 & N375S $\times 1 /$ Asn to Ser \\
\hline $\begin{array}{l}\text { Squamous Cell Ca of } \\
\mathrm{H} \& \mathrm{~N} \text { and Cervix } \\
\mathrm{n}=6 \\
\% \text { mutated: } 6.3 \%\end{array}$ & & $\begin{array}{l}\text { Median age: } 57 \\
\text { Avg age: } 58 \\
\text { M/F: } 5 / 1\end{array}$ & $\begin{array}{l}\text { IV: } 4 \\
\text { N/A: } 2\end{array}$ & $\begin{array}{c}2 \\
14 \\
14\end{array}$ & $\begin{array}{l}\text { N375S } \times 2 / \text { Asn to Ser } \\
\text { R988C } \times 2 / \text { Arg to Cys } \\
\text { T1010I } \times 2 / \text { Thr to Ile }\end{array}$ \\
\hline $\begin{array}{l}\text { Renal Cell Ca } \\
\mathrm{n}=1 \\
\text { mutated: } 25 \%\end{array}$ & & $65 \mathrm{y} / \mathrm{o} \mathrm{M}$ & IV & 2 & N375S $\times 1 /$ Asn to Ser \\
\hline
\end{tabular}

Contd... 


\begin{tabular}{|c|c|c|c|c|c|}
\hline $\begin{array}{l}\text { Tumor type (with } \\
\text { c-MET mutation) }\end{array}$ & $\begin{array}{l}\text { Other tumors in } \\
\text { these patients }\end{array}$ & $\begin{array}{l}\text { Patients' age/ } \\
\text { sex }\end{array}$ & $\begin{array}{l}\text { Tumor } \\
\text { stage }\end{array}$ & $\begin{array}{c}\text { Mutation } \\
\text { exon (s) }\end{array}$ & $\begin{array}{l}\text { Mutation codon (s)/aa } \\
\text { change (s) }\end{array}$ \\
\hline $\begin{array}{l}\text { Pheochromocytoma } \\
\text { \& Composite } \\
\text { Pheochromocytoma } \\
\mathrm{n}=2 \\
\% \text { mutated: } 100 \%\end{array}$ & & $71 \mathrm{~F} \& 48 \mathrm{y} / \mathrm{o} \mathrm{F}$ & IV & $\begin{array}{c}2 \\
14\end{array}$ & $\begin{array}{l}\text { N375S } \times 1 / \text { Asn to Ser } \\
\text { R988C } \times 1 / \text { Arg to Cys }\end{array}$ \\
\hline $\begin{array}{l}\text { Ovarian Serous } \mathrm{Ca} \\
\mathrm{n}=4 \\
\% \text { mutated: } 8.3 \%\end{array}$ & Breast $\mathrm{Ca}$ & $\begin{array}{l}\text { Median age: } 56 \\
\text { Avg age: } 56.2 \\
4 \mathrm{~F} \\
\end{array}$ & $\begin{array}{l}\text { III: } 3 \\
\text { IV: } 1\end{array}$ & $\begin{array}{c}2 \\
14\end{array}$ & $\begin{array}{l}\text { N375S } \times 2 / \text { Asn to Ser } \\
\text { T1010I } \times 2 / \text { Thr to Ile }\end{array}$ \\
\hline $\begin{array}{l}\text { Ovarian Clear Cell } \mathrm{Ca} \\
\mathrm{n}=1 \\
\% \text { mutated: } 11 \%\end{array}$ & $\mathrm{BCC}$ & $53 \mathrm{y} / \mathrm{o} \mathrm{F}$ & IV & 2 & $\mathrm{~N} 375 \mathrm{~S} \times 1 /$ Asn to Ser \\
\hline $\begin{array}{l}\text { Ovarian Mixed } \mathrm{Ca} \\
\mathrm{n}=1 \\
\% \text { mutated: } 50 \%\end{array}$ & & $61 \mathrm{y} / \mathrm{o} \mathrm{F}$ & $\mathrm{N} / \mathrm{A}$ & 14 & T1010I $\times 1 /$ Thr to Ile \\
\hline $\begin{array}{l}\text { Peritoneal Serous } \mathrm{Ca} \\
\mathrm{n}=1 \\
\% \text { mutated: } 50 \%\end{array}$ & & $56 \mathrm{y} / \mathrm{o} \mathrm{F}$ & III & 14 & T1010I $\times 1 /$ Thr to Ile \\
\hline $\begin{array}{l}\text { Breast Ductal Adenoca. } \\
\mathrm{n}=12 \\
\% \text { mutated: } 20 \%\end{array}$ & & $\begin{array}{l}\text { Median age: } 55 \\
\text { Avg age: } 55.3 \\
12 \mathrm{~F}\end{array}$ & $\begin{array}{l}\text { IV: } 7 \\
\text { III: } 1 \\
\text { II: } 1 \\
\text { N/A: } 3\end{array}$ & $\begin{array}{c}2 \\
14\end{array}$ & $\begin{array}{l}\text { N375S } \times 9 / \text { Asn to Ser } \\
\text { T1010I } \times 3 / \text { Thr to Ile }\end{array}$ \\
\hline $\begin{array}{l}\text { Uterine } \\
\text { Leiomyosarcoma } \\
\mathrm{n}=2 \\
\% \text { mutated: } 22 \%\end{array}$ & & $\begin{array}{l}54 \mathrm{y} / \mathrm{o} \mathrm{F} \& \\
56 \mathrm{y} / \mathrm{o} \mathrm{F}\end{array}$ & $\begin{array}{l}\text { IV: } 1 \\
\text { I: } 1\end{array}$ & $\begin{array}{c}2 \\
14\end{array}$ & $\begin{array}{l}\text { N375S } \times 1 / \text { Asn to Ser } \\
\text { T1010I } \times 1 / \text { Thr to Ile }\end{array}$ \\
\hline $\begin{array}{l}\text { Uterine Endometrioid } \\
\text { Adenoca. } \\
\mathrm{n}=3 \\
\% \text { mutated: } 13 \%\end{array}$ & $\begin{array}{l}\text { Breast Ca. } \times 1 \\
\text { Colonic Adenoca. } \times 1\end{array}$ & $\begin{array}{l}\text { Median age: } 63 \\
\text { Avg age: } 66.6 \\
3 \mathrm{~F}\end{array}$ & $\begin{array}{l}\text { IV: } 2 \\
\text { II: } 1\end{array}$ & $\begin{array}{c}2 \\
14\end{array}$ & $\begin{array}{l}\text { N375S } \times 2 / \text { Asn to Ser } \\
\text { T1010I } \times 1 / \text { Thr to Ile }\end{array}$ \\
\hline $\begin{array}{l}\text { Uterine MMMT } \\
\mathrm{n}=1 \\
\% \text { mutated: } 25 \%\end{array}$ & & $59 \mathrm{y} / \mathrm{o} \mathrm{F}$ & $\mathrm{N} / \mathrm{A}$ & 2 & $\mathrm{~N} 375 \mathrm{~S} \times 1 /$ Asn to Ser \\
\hline $\begin{array}{l}\text { Glioblastoma } \\
\mathrm{n}=5 \\
\% \text { mutated: } 16 \%\end{array}$ & $\begin{array}{l}\mathrm{AML} \times 1 \\
\mathrm{BCC} \times 1\end{array}$ & $\begin{array}{l}\text { Median: } 59 \text { y/o } \\
\text { Avg age: } 56.6 \\
\text { M/F: } 3 / 2\end{array}$ & & 2 & $\mathrm{~N} 375 \mathrm{~S} \times 5 /$ Asn to Ser \\
\hline $\begin{array}{l}\text { Anaplastic Glioma } \\
\mathrm{n}=2 \\
\% \text { mutated: } 66 \%\end{array}$ & & $\begin{array}{l}42 \mathrm{y} / \mathrm{o} M \& \\
45 \mathrm{y} / \mathrm{o} \mathrm{M}\end{array}$ & & 2 & N375S $\times 2 /$ Asn to Ser \\
\hline $\begin{array}{l}\text { Oligodendroglioma } \\
\mathrm{n}=1 \\
\% \text { mutated: } 50 \%\end{array}$ & & 33 y/o F & & 14 & T1010I $\times 1 /$ Thr to Ile \\
\hline $\begin{array}{l}\text { Desmoplastic Small } \\
\text { Round Cell Tumor } \\
\mathrm{n}=1 \\
\% \text { mutated: } 14 \%\end{array}$ & & $17 \mathrm{y} / \mathrm{o} \mathrm{M}$ & $\mathrm{N} / \mathrm{A}$ & 2 & $\mathrm{~N} 375 \mathrm{~S} \times 1 /$ Asn to Ser \\
\hline $\begin{array}{l}\text { Squamous Cell Ca of } \\
\text { Rectum } n=1 \text { mutated: } \\
25 \%\end{array}$ & & $\mathrm{~N} / \mathrm{A}$ & $\mathrm{N} / \mathrm{A}$ & 2 & N375S $\times 1 /$ Asn to Ser \\
\hline
\end{tabular}

Contd... 


\begin{tabular}{|c|c|c|c|c|c|}
\hline $\begin{array}{l}\text { Tumor type (with } \\
\text { c-MET mutation) }\end{array}$ & $\begin{array}{l}\text { Other tumors in } \\
\text { these patients }\end{array}$ & $\begin{array}{l}\text { Patients' age/ } \\
\text { sex }\end{array}$ & $\begin{array}{l}\text { Tumor } \\
\text { stage }\end{array}$ & $\begin{array}{l}\text { Mutation } \\
\text { exon (s) }\end{array}$ & $\begin{array}{l}\text { Mutation codon (s)/aa } \\
\text { change (s) }\end{array}$ \\
\hline $\begin{array}{l}\text { Salivary Gland } \mathrm{Ca} \\
\mathrm{n}=2 \\
\% \text { mutated: } 10 \%\end{array}$ & $\mathrm{PTC} \times 1$ & $\begin{array}{l}48 \text { y/o F \& } \\
73 \text { y/o M }\end{array}$ & $\begin{array}{l}\text { IV: } 1 \\
\text { N/A: } 1\end{array}$ & 2 & N375S $\times 2 /$ Asn to Ser \\
\hline $\begin{array}{l}\text { Heart Angiosarcoma } \\
\mathrm{n}=2 \\
\% \text { mutated: } 50 \%\end{array}$ & & $\begin{array}{l}59 \mathrm{y} / \mathrm{o} \mathrm{M} \mathrm{\&} \\
31 \mathrm{y} / \mathrm{o} \mathrm{F}\end{array}$ & $\mathrm{N} / \mathrm{A}$ & $\begin{array}{c}2 \\
14\end{array}$ & $\begin{array}{l}\text { N375S } \times 1 / \text { Asn to Ser } \\
\text { T1010I } \times 1 / \text { Thr to Ile }\end{array}$ \\
\hline $\begin{array}{l}\text { GIST } \\
\mathrm{n}=2 \\
\% \text { mutated: N/A }\end{array}$ & & $\begin{array}{l}57 \mathrm{y} / \mathrm{o} \mathrm{M} \mathrm{\&} \\
67 \mathrm{y} / \mathrm{o} \mathrm{M}\end{array}$ & $\begin{array}{l}\text { IV: } 1 \\
\text { N/A: } 1\end{array}$ & $\begin{array}{l}14 \\
14\end{array}$ & $\begin{array}{l}\text { T1010I } \times 1 / \text { Thr to Ile } \\
\text { R988C } \times 1 / \text { Arg to Cys }\end{array}$ \\
\hline $\begin{array}{l}\text { Invasive Thymoma } \\
\mathrm{n}=1 \\
\% \text { mutated: } 50 \%\end{array}$ & & $\mathrm{~N} / \mathrm{A}$ & IV & 2 & N375S $\times 1 /$ Asn to Ser \\
\hline $\begin{array}{l}\text { Spindle Sarcoma NOS } \\
\mathrm{n}=2 \\
\% \text { mutated: } 16 \%\end{array}$ & & $\begin{array}{l}61 \mathrm{y} / \mathrm{o} \mathrm{M} \mathrm{\&} \\
69 \mathrm{y} / \mathrm{o} \mathrm{M}\end{array}$ & N/A & 2 & N375S $\times 2 /$ Asn to Ser \\
\hline $\begin{array}{l}\text { Malignant } \\
\text { Mesothelioma } \\
\mathrm{n}=1 \\
\% \text { mutated: } 33 \%\end{array}$ & & 59 y/o M & $\mathrm{N} / \mathrm{A}$ & 2 & N375S $\times 1 /$ Asn to Ser \\
\hline
\end{tabular}

Abbreviations - y/o: year old, avg - Average, M - Male, F - Female, Adenoca - Adenocarcinoma, Ca - Carcinoma, SCC - Squamous cell carcinoma, BCC - Basal cell carcinoma, PTC - Papillary thyroid cancer, AML - Acute myeloid leukemia, MMMT - Malignant mixed mullerian tumor, GIST - Gastrointestinal stromal tumor, N/A - Not available

\begin{tabular}{|l|c|c|c|c|c|}
\hline \multicolumn{6}{|l|}{ Table 2: Summary of incidence of c-MET point mutations with corresponding co-mutations in the studied tumors } \\
\hline Co-Mutations & N375S & R988C & T1010I & Y1248H & Y1253D \\
\hline P53 & 4 & 0 & 4 & 0 & 1 \\
\hline KRAS & 13 & 5 & 9 & 0 & 1 \\
\hline NRAS & 3 & 2 & 0 & 0 & 0 \\
\hline BRAF & 8 & 2 & 3 & 0 & 0 \\
\hline PIK3CA & 5 & 3 & 1 & 0 & 0 \\
\hline IDH1 & 1 & 0 & 1 & 0 & 0 \\
\hline EGFR & 1 & 0 & 0 & 0 & 0 \\
\hline AKT & 0 & 0 & 2 & 0 & 0 \\
\hline KIT & 0 & 1 & 1 & 0 & 0 \\
\hline
\end{tabular}

mutated genes included p53, KRAS, NRAS, BRAF, PIK3CA, IDH1 KIT, AKT and EGFR, the distribution of which corresponded to trends generally associated with each tumor type. Save for the one case with Y1253D aberration and co-mutations $(1 / 1=100 \%)$, the highest rate of co-mutations was observed with R988C aberration, corresponding to $76 \%$ (10/13). The next highest co-mutation rate was with T1010I, corresponding to $37 \%(14 / 38)$ closely followed by N375S at $35 \%(29 / 81)$. No co-mutation was clustered with codon Y1248H. This patient also had history of squamous cell carcinoma and prostatic adenocarcinoma.

In cases with metastatic, surgically resected colorectal cancer (stages III/IV), the following mutations and percentages were detected: c-MET 9.5\%, KRAS 43\%, PIK3CA $18 \%$, NRAS $4 \%$, and BRAF $8 \%$. The remaining mutations included P53, EGFR, KIT, and IDH mutations. $3 \%$ of tumors had no mutation detected on analysis. Neither the c-MET mutated nor the subset of cases with no mutation detected showed overexpression of c-MET by FISH, and targeted c-MET treatment was not utilized in their treatment. Those whose sole mutation detected on the panel was a c-MET mutation $(\mathrm{n}=14)$ had a better survival compared to subjects with no mutations detected in the analysis $(n=24)$. However, this difference was only marginally significant $(p=0.057)$, which may be due to limited sample size. Results are summarized in tables 1-3 and Figure 1 (Kaplan Meier 


\begin{tabular}{|l|l|l|}
\hline Table 3: Summary of tumor types and their corresponding c-MET point mutations and co-mutations \\
\hline Tumor type (s) & c-MET Mutation (s) & Co-mutation (s) \\
\hline Colorectal Adenocarcinoma & $\begin{array}{l}\text { N375S, R988C, T1010I, } \\
\text { Y1248H, Y1253D }\end{array}$ & $\begin{array}{l}\text { P53, KRAS, PI3K, BRAF, IDH1, } \\
\text { AKT }\end{array}$ \\
\hline Melanoma & N375S, T1010I, R988C & NRAS, BRAF \\
\hline Lung Adenocarcinoma & N375S, R988C, T1010I & EGFR, KRAS, BRAF, P53, NRAS \\
\hline Thyroid Carcinoma & N375S (PTC), T1010I (Medullary carcinoma)* & BRAF, PI3K, P53* \\
\hline Ovarian Carcinoma & N375S, T1010I & P53, KRAS \\
\hline Uterine Endometriod Carcinoma & T1010I, N375S & AKT, KRAS \\
\hline Breast Adenocarcinoma & N375S & PI3K \\
\hline Squamous Cell Carcinoma & N375S, R988C, T1010I & PI3K \\
\hline Prostatic Adenocarcinoma & N375S & P53 \\
\hline Appendiceal Adenocarcinoma & N375S, R988C & KRAS \\
\hline $\begin{array}{l}\text { Duodenal \& Pancreatic } \\
\text { Adenocarcinoma }\end{array}$ & N375S & KRAS \\
\hline Heart Angiosarcoma & T1010I, N375S & KRAS \\
\hline Gastrointestinal Stromal Tumor & T1010I, R988C & KIT \\
\hline Oligodendroglioma & T1010I & IDH1 \\
\hline
\end{tabular}

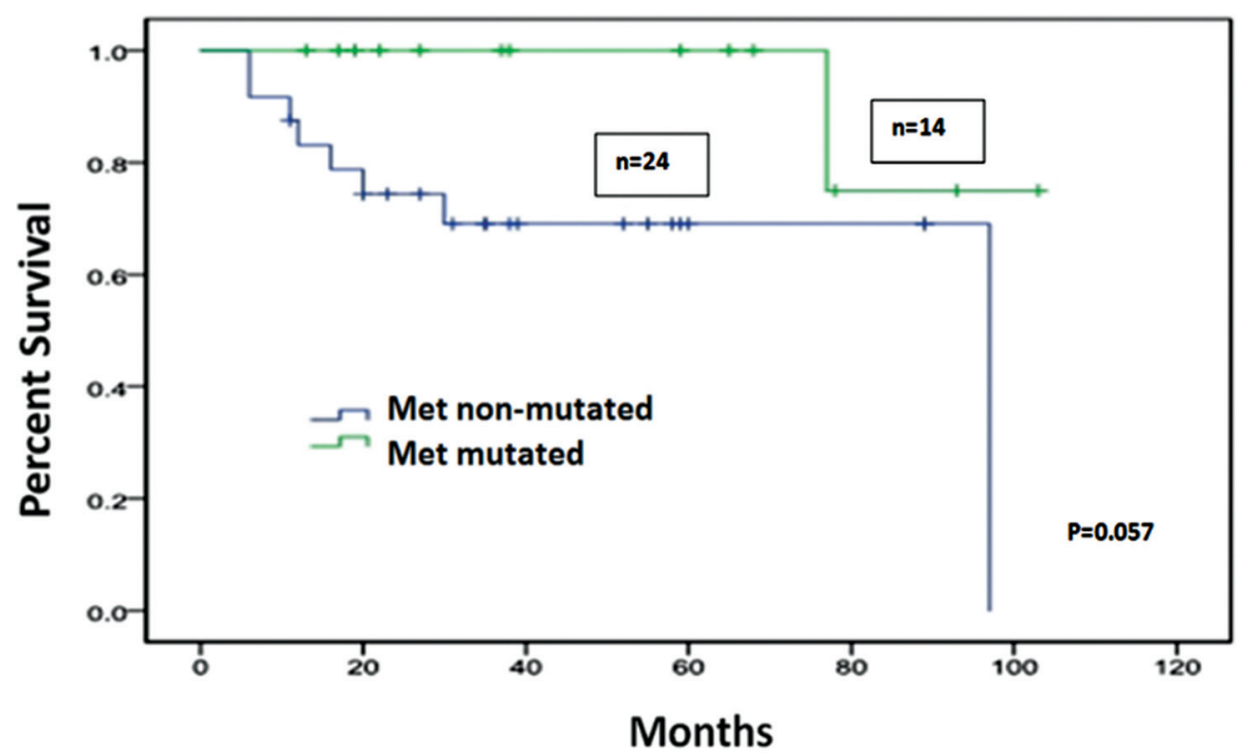

Figure 1: Kaplan Meier Graph of survival comparision between patients with metastatic CRC and only c-MET mutation vs. those who no mutation detected on the molecular test panel

curve). In cases with metastatic CRC, logistic regression analysis of age and gender against c-MET mutational status was negative for any significant association (p: 0.88 and p: 0.56 respectively).

\section{DISCUSSION}

C-MET receptor tyrosine kinase and its physiological ligands $\mathrm{HGF} / \mathrm{SF}$ are associated with diverse cellular response upon activation. Ligand mediated dimerization triggers phosphorylation of c-MET and response of cytoplasmic effector proteins including GAB1, GRB2, phospholipase $\mathrm{C}$ and SRC. C-MET is intimately associated with pathways including RAS-MAPK, PI3K-AKT and Wnt [5].

The role of c-MET in tumorigenesis through genetic aberration, up regulation of cellular cross talk, and roles in metastasis and activation of cancer stem cells is an area of ongoing research. The evidence for it playing a role in oncogenesis is mounting as not only its somatic mutations 
have been found in many cancers, germ line c-MET point mutations are present in subset of tumors such as papillary renal cell carcinoma. In parallel a subset of genetic mutations are found as polymorphic variants. Although in our study we did not find upregulation in MET gene expression by FISH analysis, we did detect missense point mutations that through their altered gene product can change the functionality of the c-MET receptor.

The most commonly identified mutation in our series was the N375S of the extracellular SEMA domain. This aberration results in the medium sized polar amino acid asparagine being replaced by the smaller polar amino acid serine and has been found as a germ line mutation in a cohort of patients with non-small cell lung cancer (NSCLC) [6]. A later study found this mutation at a higher rate in the normal healthy controls than in the tumors indicating is a germline polymorphism rather than a cancer driving aberration [7]. Similar findings are indicated by Greenman et. al. to find N375S as a passenger and not a driver mutation [8]. These studies clearly cast doubt as to its role in tumorogenesis. The N375S mutation has been found to result in a c-MET receptor which is resistant to inhibition [6]. In comparison, the T1010I mutation is found a more robust target for chemotherapy $[9,10]$. It is plausible that in a tumor harboring concurrent c-MET aberrations, such as the one melanoma case in our review, the N375S mutation may hinder the efficacy of the c-MET directed treatment. As chemotherapy becomes more sophisticated and personalized medicine gains reality, the implications of mutations such as the N375S mutation can become more relevant in establishing targeted treatments.

Despite evidence supporting the T1010I mutation's importance in oncogenesis, not every study confirms this role. This mutation, in the cytoplasmic juxtamembrane domain, results in the substitution of the medium sized hydrophobic amino acid threonine with the medium sized polar amino acid isoleucine. It has been shown to increase growth factor independent proliferation and motility in vitro in tumor cell lines [9]. Wasenius et. al. found this mutation more frequently in thyroid carcinomas than in the goiter controls [10]. Interestingly, 4 of the 6 mutations were also found to be germline. Yet its significance has been challenged by other studies which report a low incidence of T1010I mutation in both tumors and controls and not resulting in an enhanced c-MET phosphorylation $[9,11]$.

The other c-MET mutation R988C, affecting the cytoplasmic juxtamembrane domain, results in the substitution of the large polar amino acid arginine with the small polar amino acid cysteine. Studies on this mutation are also conflicting as some demonstrate it resulting in gain a function in SCLC cell lines $[9,12]$, while others report its lack of transforming oncogenic capacity and therefore of little significance $[11,13]$.

The two remaining mutations detected are both affecting the kinase domain. The Y1248H mutation results in the substitution of the large non-polar aromatic amino acid tyrosine with the medium sized polar amino acid histidine. It is a known mutation found in papillary renal cell carcinoma and reported to have a transforming ability $[14,15]$. This mutation is relevant in chemotherapeutic targeting and has been found resistant to the c-MET inhibitor SU11274 [16]. The Y1253D mutation results in the substitution of the large non-polar aromatic amino acid tyrosine for the medium sized polar amino acid aspartic acid. This mutation has been found in nodal metastases of head and neck carcinoma and when introduced into a cell line resulted in a more motile and invasive cell phenotype [17].

The discrepancies between studies on whether a particular mutation is transformative may be explained if the same aberration results in different cell phenotypes when affecting different cell lineages. To support this notion, a recent study indicates that overexpression of c-MET associated with a worse prognosis in esophageal adenocarcinoma and not in head and neck cancers [18]. Despite the controversies regarding functionality of c-MET mutations, more recent and uniformly reported observations are that c-MET mutation in conjunction with other mutations likely confers a more aggressive tumor profile and that c-MET aberration is more prevalent in metastatic/invasive foci compared to the primary tumor $[17,18]$.

Polymorphisms of c-MET which on their own may lack transformative ability may still play a role in promotion of tumor development and metastasis in combination with other mutations. Its relevance may be closely tied to comutation rather than the sole effect of this mutation alone. According to Lee et al., activated c-MET has been shown to be a predictor of poor survival when in combination with other mutations in CRC. The authors identified that clustering of activated c-MET in conjunction with PIK3CA mutation predicts a worse survival outcome [19]. The effect of these mutations may depend on whether evolved in germ line or somatic cells, the paracrine milieu in addition to the presence of secondary co-mutations.

As stated previously, a subset of c-MET aberrations can play a role in response to chemotherapy by rendering the receptors resistant to drug therapies targeting c-MET. This would clearly effect the treatment response by decreasing the response to the drug. In EGFR mutated NSCLC, MET amplification has been demonstrated as an escape pathway and a mechanism of EGFR TKI resistance [20]. Also, in patients with c-MET amplified NSCLC, addition of a c-MET inhibitor has been shown to lead to improvement of survival [21]. These findings make it likely that MET inhibitors will be an important addition to combination chemotherapy and also more robust tumor profiling, an important part of patient care.

Current literature empirically suggests that c-MET aberrations (mutations/protein over expression) are more commonly noted in tumor clones with potential to spread. Activating c-MET aberrations have been found selected in association with nodal metastasis or invasive fronds of the primary tumors. This increased prevalence has been theorized to involve c-MET's signaling function in angiogenesis and 
cell movement $[6,20,21]$. A higher than expected range of c-MET mutations noted in our review is perhaps in parallel with the notion of clustering of c-MET with metastatic fronds, as greater than $95 \%$ of cases in our study were of metastatic tumors.

Diversity of proposed neoplastic mechanisms has led to producing a wealth of inhibitors targeting various aspects of the c-MET pathway. Currently clinical trials are underway involving c-MET and cancers including NSCLC, hepatocellular carcinoma, renal cell carcinoma, cholangiocarcinoma, triple negative breast adenocarcinoma, gastric or esophageal adenocarcinoma, and anaplastic large cell lymphoma, to name a few (http://clinicaltrials.gov/ ct2/results?term=met). Most of these trials investigate the effects of c-MET inhibitors, usually in combination with other tyrosine kinase inhibitors or other chemotherapy drugs.

Our study did not find a survival disadvantage for patients with resected metastatic CRC harboring only c-MET mutation compared to those with none, but rather a marginally significant survival improvement. Although the small study population may limit analysis, we did not find a clear-cut adverse prognostic relevance attributable to c-MET mutation alone, when unaccompanied by a known oncogenic mutation. Further studies may aid in establishing this concept.

\section{ACKNOWLEDGEMENTS}

Not applicable

\section{CONFLICT OF INTEREST}

The authors have nothing to disclose.

\section{GRANT SUPPORT}

Not applicable.

\section{REFERENCES}

1. Peruzzi B. Targeting the c-Met Signaling Pathway in Cancer. Clin Cancer Res. 2006 Jun 15;12(12):3657-60.

2. Ma PC. Functional Expression and Mutations of c-Met and Its Therapeutic Inhibition with SU11274 and Small Interfering RNA in Non-Small Cell Lung Cancer. Cancer Res. 2005 Feb 15;65(4):1479-88.

3. Gherardi E, Youles ME, Miguel RN, Blundell TL, Iamele L, Gough J, et al. Functional map and domain structure of MET, the product of the c-met protooncogene and receptor for hepatocyte growth factor/scatter factor. Proc Natl Acad Sci. 2003;100(21):12039-44.

4. Wickramasinghe D, Kong-Beltran M. Met Activation and Receptor Dimerization in Cancer: A Role for the Sema Domain. Cell Cycle. 2005 Mar 2;4(5):683-5.
5. Gherardi E, Birchmeier W, Birchmeier C, Woude GV. Targeting MET in cancer: rationale and progress. Nat Rev Cancer. 2012 Jan 24;12(2):89-103.

6. Krishnaswamy S, Kanteti R, Duke-Cohan JS, Loganathan S, Liu W, Ma PC, et al. Ethnic differences and functional analysis of MET mutations in lung cancer. Clin Cancer Res Off J Am Assoc Cancer Res. 2009 Sep 15;15(18):5714-23.

7. Tengs T, Lee JC, Guillermo Paez J, Zhao X, LaFramboise T, Giannoukos G, et al. A transforming MET mutation discovered in non-small cell lung cancer using microarraybased resequencing. Cancer Lett. 2006 Aug;239(2):227-33.

8. Greenman C, Stephens P, Smith R, Dalgliesh GL, Hunter C, Bignell G, et al. Patterns of somatic mutation in human cancer genomes. Nature. 2007 Mar 8;446(7132):153-8.

9. Ma PC, Kijima T, Maulik G, Fox EA, Sattler M, Griffin JD, et al. c-MET Mutational Analysis in Small Cell Lung Cancer Novel Juxtamembrane Domain Mutations Regulating Cytoskeletal Functions. Cancer Res. 2003;63(19):6272-81.

10. Wasenius V-M, Hemmer S, Karjalainen-Lindsberg M-L, Nupponen NN, Franssila K, Joensuu H. MET receptor tyrosine kinase sequence alterations in differentiated thyroid carcinoma. Am J Surg Pathol. 2005 Apr;29(4):544-9.

11. Voortman J, Harada T, Chang RP, Killian JK, Suuriniemi M, Smith WI, et al. Detection and therapeutic implications of c-Met mutations in small cell lung cancer and neuroendocrine tumors. Curr Pharm Des. 2013;19(5):833-40.

12. Jagadeeswaran R, Jagadeeswaran S, Bindokas VP, Salgia R. Activation of $\mathrm{HGF} / \mathrm{c}-$ Met pathway contributes to the reactive oxygen species generation and motility of small cell lung cancer cells. Am J Physiol Lung Cell Mol Physiol. 2007 Jun;292(6):L1488-94.

13. Tyner JW, Fletcher LB, Wang EQ, Yang WF, RutenbergSchoenberg ML, Beadling C, et al. MET Receptor Sequence Variants R970C and T992I Lack Transforming Capacity. Cancer Res. 2010 Aug 1;70(15):6233-7.

14. Schmidt L, Junker K, Nakaigawa N, Kinjerski T, Weirich G, Miller M, et al. Novel mutations of the MET proto-oncogene in papillary renal carcinomas. Oncogene. 1999 Apr 8;18(14):2343-50.

15. Jeffers M, Schmidt L, Nakaigawa N, Webb CP, Weirich G, Kishida $\mathrm{T}$, et al. Activating mutations for the met tyrosine kinase receptor in human cancer. Proc Natl Acad Sci U S A. 1997 Oct 14;94(21):11445-50.

16. Berthou S, Aebersold DM, Schmidt LS, Stroka D, Heigl C, Streit B, et al. The Met kinase inhibitor SU11274 exhibits a selective inhibition pattern toward different receptor mutated variants. Oncogene. 2004 Apr 5;23(31):5387-93.

17. Lorenzato A, Olivero M, Patanè S, Rosso E, Oliaro A, Comoglio PM, et al. Novel somatic mutations of the MET oncogene in human carcinoma metastases activating cell motility and invasion. Cancer Res. 2002 Dec 1;62(23):7025-30.

18. Mesteri I, Schoppmann SF, Preusser M, Birner P. Overexpression of CMET is associated with signal transducer and activator of transcription 3 activation and diminished prognosis in oesophageal adenocarcinoma but not in squamous cell carcinoma. Eur J Cancer Oxf Engl 1990. 2014 May;50(7):1354-60.

19. Lee J, Jain A, Kim P, Lee T, Kuller A, Princen F, et al. 
Activated cMET and IGF1R-driven PI3K signaling predicts poor survival in colorectal cancers independent of KRAS mutational status. PloS One. 2014;9(8):e103551.

20. Engelman JA, Zejnullahu K, Mitsudomi T, Song Y, Hyland C, Park JO, et al. MET amplification leads to gefitinib resistance in lung cancer by activating ERBB3 signaling. Science.
2007 May 18;316(5827):1039-43.

21. SpigelDR, Ervin TJ, Ramlau RA, DanielDB, GoldschmidtJH, Blumenschein GR, et al. Randomized phase II trial of Onartuzumab in combination with erlotinib in patients with advanced non-small-cell lung cancer. J Clin Oncol Off J Am Soc Clin Oncol. 2013 Nov 10;31(32):4105-14. 